\title{
THE FATE OF SPIRAL GALAXIES IN CLUSTERS: THE STAR FORMATION HISTORY OF THE ANEMIC VIRGO CLUSTER GALAXY NGC 4569
}

\author{
A. Boselli, ${ }^{1}$ S. Boissier, ${ }^{1,2}$ L. Cortese, ${ }^{1,3}$ A. Gil de Paz, ${ }^{2,4}$ M. Seibert, ${ }^{5}$ B. F. Madore, $, 2,6$ \\ V. Buat, ${ }^{1}$ and D. C. Martin ${ }^{5}$ \\ Received 2006 June 8; accepted 2006 July 13
}

\begin{abstract}
We present a new method for studying the star formation history of late-type cluster galaxies undergoing gas starvation or a ram pressure stripping event by combining bidimensional multifrequency observations with multizone models of galactic chemical and spectrophotometric evolution. This method is applied to the Virgo Cluster anemic galaxy NGC 4569. We extract radial profiles from recently obtained UV GALEX images at 1530 and $2310 \AA$, from visible and near-IR narrow ( $\mathrm{H} \alpha)$ and broadband images at different wavelengths $(u, B, g, V, r, i, z, J, H$, and $K)$, from Spitzer IRAC and MIPS images, and from atomic and molecular gas maps. The model in the absence of interaction (characterized by its rotation velocity and spin parameter) is constrained by the unperturbed $H$-band light profile and by the $\mathrm{H} \alpha$ rotation curve. We can reconstruct the observed total gas radial density profile and the light surface brightness profiles at all wavelengths in a ram pressure stripping scenario by making simple assumptions about the gas removal process and the orbit of NGC 4569 inside the cluster. The observed profiles cannot be reproduced by simply stopping gas infall, thus mimicking starvation. Gas removal is required, which is more efficient in the outer disk, inducing radial quenching in the star formation activity, as observed and reproduced by the model. This observational result, consistent with theoretical predictions that a galaxy cluster-IGM interaction is able to modify structural disk parameters without gravitational perturbations, is discussed in the framework of the origin of lenticular galaxies in clusters.
\end{abstract}

Subject headings: galaxies: clusters: individual (Virgo) — galaxies: individual (NGC 4569-M90) — galaxies: interactions - ultraviolet: galaxies

Online material: color figures

\section{INTRODUCTION}

Perturbations induced by the harsh cluster environment make the evolution of cluster galaxies different from that of their counterparts in the field. Besides the well-known morphology segregation effect (Dressler 1980; Whitmore et al. 1993), there is also evidence clearly indicating that the cluster late-type galaxy population is systematically different from the field: cluster spiral galaxies are generally depleted in their total $\mathrm{H}$ i content and are less active in forming stars than their isolated counterparts. This is most recently and extensively reviewed in Boselli \& Gavazzi (2006). The nature of the perturbing phenomenon has not yet been unambiguously identified. Several physical processes have been proposed to explain gas removal in clusters. Some of them are related to the dynamical interactions of cluster galaxies with

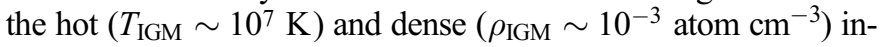
tergalactic medium (IGM) (ram pressure, Gunn \& Gott 1972; viscous stripping, Nulsen 1982; thermal evaporation, Cowie \& Songaila 1977). Others are due to the gravitational interactions with nearby companions (Merritt 1983) or with the wider po-

\footnotetext{
${ }^{1}$ Laboratoire d'Astrophysique de Marseille, BP8, Traverse du Siphon, F-13376 Marseille, France.

${ }^{2}$ Observatories of the Carnegie Institution of Washington, 813 Santa Barbara Street, Pasadena, CA 91101.

3 School of Physics and Astronomy, Cardiff University, 5, The Parade, Cardiff CF24 3YB, UK.

${ }^{4}$ Departamento de Astrofísica y CC. de la Atmósfera, Universidad Complutense de Madrid, Avenida de la Complutense, s/n, E-28040 Madrid, Spain.

${ }^{5}$ California Institute of Technology, Mail Code 405-47, 1200 East California Boulevard, Pasadena, CA 91125.

${ }^{6}$ NASA/IPAC Extragalactic Database, California Institute of Technology, Mail Code 100-22, 770 South Wilson Avenue, Pasadena, CA 91125.
}

tential of the cluster (Byrd \& Valtonen 1990). The detailed analyses of several well-known resolved galaxies in the Virgo and Coma Clusters generally favor a galaxy-IGM interaction scenario (Vollmer et al. 1999, 2000, 2001a, 2004b; Kenney et al. 2004; Yoshida et al. 2004) in these instances. Using complete, multifrequency data sets combined with model predictions, several studies have unambiguously shown that ram pressure stripping occurs even at the periphery of clusters (e.g., CGCG 97-73 and 97-79 in A1367; Gavazzi et al. 2001) and that galaxies recently ( $\leq 500 \mathrm{Myr}$ ) stripped by ram pressure populate nearby clusters well outside the cluster core (Boselli \& Gavazzi 2006).

Ram pressure stripping has also been invoked to explain the origin of the lenticular galaxy population inhabiting rich clusters. One early suggestion was that, as a consequence of gas removal through ram pressure stripping, cluster galaxies first become "anemic" and then passively evolved into lenticular galaxies (van den Bergh 1976). Statistical considerations, as well as structural, kinematical, and spectroscopic properties of lenticular galaxies, however, do not appear to confirm this simple scenario. As discussed in Dressler $(1980,2004)$, the morphological type variation in clusters is too weak a function of the local galaxy density. Furthermore, the disks of lenticular galaxies have, on average, surface brightnesses and bulge-to-disk ratios that are significantly higher than early-type spiral galaxies (Dressler 1980; Christlein \& Zabuldoff 2004; Boselli \& Gavazzi 2006), making the formation of lenticular galaxies through gas sweeping in spiral galaxies very unlikely. The larger scatter and a small zero-point offset in the Tully-Fisher relation observed in the Virgo and Coma Cluster lenticular galaxies also indicate that $\mathrm{S} 0$ galaxies can hardly be formed by simple gas removal from healthy spiral galaxies (Hinz et al. 2003). Furthermore, cluster lenticular galaxies generally have spectroscopic 


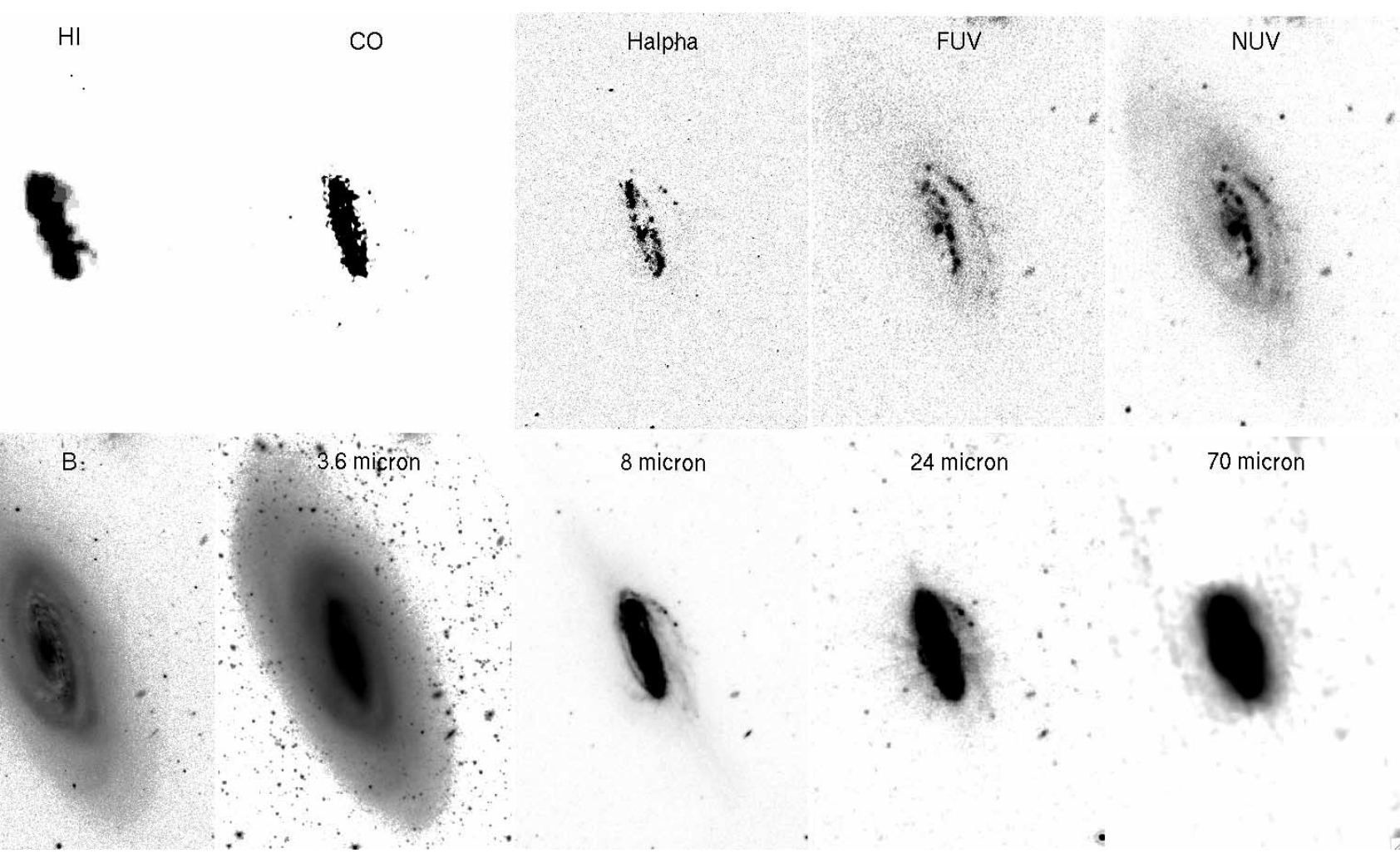

Fig. 1.-Multifrequency images of NGC 4569 at their intrinsic resolution: from top left to bottom right, $\mathrm{H}$ I, CO, $\mathrm{H} \alpha$, FUV, near-ultraviolet (NUV), $B$; and 3.6, 8, 24, and $70 \mu \mathrm{m}$ are all on the same scale.

signatures of recent, poststarburst activity (Poggianti et al. 2004) difficult to trigger in a simple ram pressure stripping scenario.

The origin of lenticular galaxies is still an open problem and limits our understanding of the evolution of galaxies in different environments. This riddle can be solved only by first realistically quantifying the physical effects of ram pressure stripping by combining model predictions with multifrequency observations of representative samples. We have been collecting multifrequency data for a large sample of late-type galaxies in nearby clusters and in the field in order to undertake the comparative statistical analysis of any systematic differences between cluster and field objects. The most important results obtained from the analysis done so far are reported in Boselli \& Gavazzi (2006). Combined with multizone models for the chemical and spectrophotometric evolution of galaxies (Boissier \& Prantzos 2000), this unique database is helping us to understand the evolution of cluster spiral galaxies. The aim of the present paper is to give a complete description of the multizone spectrophotometric models of galaxy evolution used to study the effects of the environment on cluster galaxies.

As a first application we present a detailed study of the radial profiles of the Virgo Cluster galaxy NGC 4569 (M90). NGC 4569, the prototype anemic galaxy as defined by van den Bergh (1976), is extremely deficient in $\mathrm{H} \mathrm{I}$, having only about one-tenth of the atomic gas of a comparable field galaxy of similar type and dimensions. This galaxy has a truncated $\mathrm{H} \alpha$ and $\mathrm{H}$ I radial density profile (at a radius of $\sim 5 \mathrm{kpc}$; see Figs. 1 and 3 ) as first noted by Koopmann \& Kenney (2004a, 2004b) and Cayatte et al. (1994), bearing witness to a recent interaction with the cluster environment. NGC 4569 is located close $\left(\sim 2^{\circ}, \sim 240 \mathrm{kpc}\right.$ for a virial radius of $1.7 \mathrm{Mpc})$ to the cluster center. Being one of the largest galaxies $\left(\sim 10^{\prime}, \sim 50 \mathrm{kpc}\right)$ in the Virgo Cluster, NGC 4569 is the ideal candidate for our study, since it is spatially resolved at all the wavelengths considered here. NGC 4569 has also been selected because it has been previously studied using dynamical models by Vollmer et al. (2004a). A direct comparison of the results obtained using totally independent techniques (in particular, dating the interaction) is thus possible. We just remind the reader that multifrequency and kinematical observations of the inner part of NGC 4569 done by Jogee et al. (2005) seem to indicate that the nucleus and the inner stellar bar of the galaxy might have been triggered by a recent tidal interaction. There are, however, no observational evidences indicating that the $\mathrm{H}$ I disk has been truncated by any gravitational interaction with nearby companions. Since our models are adapted for disk galaxies, we will, however, limit our study to the disk component excluding the central nucleus.

The combination of the multifrequency two-dimensional data with our spectrophotometric models allow us to study, for the first time, the radial evolution of the different stellar populations in this prototype, gas-stripped cluster galaxy with the aim of understanding whether its structural properties can evolve, even in principle, into those of a typical cluster lenticular (S0) galaxy. A more complete analysis of a statistically significant sample of cluster galaxies will be presented in a future communication.

\section{DATA}

The large amount of spectrophotometric data available for NGC 4569, collected in the GOLDMine database (Gavazzi et al. 2003), allow us to reconstruct its radial profile at 22 different wavelengths: from the new Galaxy Evolution Explorer (GALEX) UV bands (at FUV $=1530$ and NUV = $2310 \AA \AA$; IR 1.0 data release recently published in Gil de Paz et al. [2006]) to the visible $B$ and $V$ (Boselli et al. 2003); Sloan $u, g, r, i, z$ (Abazajian et al. 2005); near-IR $J, H$, and $K$ bands (Boselli et al. 1997; Two Micron All Sky Survey, Jarrett et al. 2003); mid-IR 3.6, 4.5, 5.8, and $8 \mu \mathrm{m}$ 
Infrared Array Camera (IRAC); and far-IR 24, 70, and $160 \mu \mathrm{m}$ Multiband Imaging Photometer for Spitzer (MIPS) bands recently obtained by Spitzer in the framework of the Spitzer Infrared Nearby Galaxies Survey project (Kennicutt et al. 2003). An accurate description of the IRAC and MIPS Spitzer data reduction procedures is given in Dale et al. (2005); $\mathrm{H} \alpha+[\mathrm{N}$ II] narrowband imaging, used to trace the recent star formation activity (e.g., Boselli et al. 2001), is available from Boselli \& Gavazzi (2002). Some of the multifrequency images of NGC 4569 are shown in Figure 1. The accuracy of photometry from the imaging data is, on average, about 10\%. H I profiles are from Cayatte et al. (1994), while $\mathrm{H}_{2}$ profiles, determined from $\mathrm{CO}$ data using a luminositydependent $\mathrm{CO}$ to $\mathrm{H}_{2}$ conversion factor (from Boselli et al. [2002], applied to the whole profile with no radial variation, as unfortunately no metallicity gradient information is available for NGC 4569), are taken from the Berkeley-Illinois-Maryland Association (BIMA) survey of Helfer et al. (2003) for the inner disk and from Kenney \& Young (1988) for the outer disk. The total gas profile is the sum of the $\mathrm{H}_{\mathrm{I}}$ and $\mathrm{H}_{2}$ gas profiles multiplied by a factor of 1.4 to take into account the He contribution. The galaxy rotation curve has been taken from Rubin et al. (1999).

The radial surface density profiles have been constructed by integrating the available images within elliptical, concentric annuli. The ellipticity and position angles have been determined and then fixed using the deepest $B$-band image following the procedure of Gavazzi et al. (2000). ${ }^{7}$ All the observed profiles have been smoothed to the same angular resolution as the models (which is $1 \mathrm{kpc}$; see $\S 3$ ). The UV to near-IR broadband radial profiles of the galaxy have been corrected for internal extinction using the recipe of Boissier et al. (2004) based on the radial variation of the far-IR to far-ultraviolet (FUV) flux ratio. After masking the contribution of the nucleus, whose emission is contaminated by active galactic nucleus activity (NGC 4569 is classified as a Seyfert in the NASA/IPAC Extragalactic Database [NED]), we combined the 24,70 , and $160 \mu \mathrm{m}$ profiles, smoothed to the $160 \mu \mathrm{m}$ resolution, using the recipe of Dale et al. (2001) to estimate the total $1-1000 \mu \mathrm{m}$ dust emission (total far-infrared [TIR]), as extensively discussed in Cortese et al. (2006b). The TIR to FUV flux ratio radial variation is then transformed into a FUV extinction gradient (in magnitudes) using the appropriate calibration given in L. Cortese et al. (2006, in preparation) for a galaxy with spectral properties similar to those of NGC 4569 . We stress that, given its quiescent nature, the contribution of FUV photons to the dust heating of NGC 4569 is only marginal: the calibration of the far-IR to FUV flux ratio into a FUV extinction is thus more indirect than in star-forming galaxies, where dust is mostly heated by UV photons, making the correction highly uncertain. The adopted far-IR to FUV flux ratio versus $A(\mathrm{FUV})$ relation calibrated on the spectral energy distribution of NGC 4569 allows us to also take into account the contribution to dust heating from the general interstellar radiation field not necessarily associated to star-forming regions. Indeed, we predict less extinction for the same TIR/FUV ratio than for star-forming galaxies (Buat et al. 2005). The extinction in the other visible and nearIR bands has been determined using the prescription of Boselli et al. (2003). The resulting $A(\mathrm{FUV})$ extinction profile, along with $A(B)$ and $A(H)$, are shown in Figure 2: given the lack of gas and dust in the outer disk, extinctions are extrapolated to zero at large radii. As extensively discussed in the next sections, the

\footnotetext{
${ }^{7}$ To avoid any possible contamination by the northwest arm, whose kinematical properties indicate that it is not associated to the stellar disk but rather formed during the galaxy cluster-IGM interaction, the arm was masked in the construction of the radial profiles. If included, its contribution would be perceptible only in the FUV filter at radii $>8 \mathrm{kpc}$, increasing the surface brightness by $<0.5 \mathrm{mag}$.
}

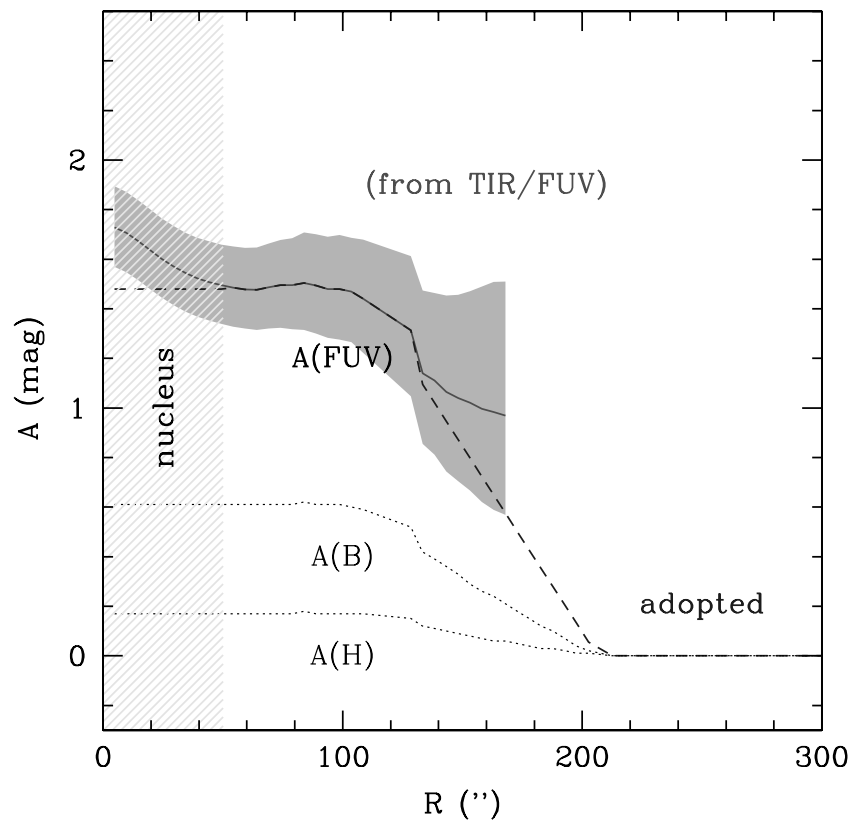

FIG. 2.- Radial variation of the $A(\mathrm{FUV})$ extinction profile (solid line) and its uncertainty (dark gray shaded area) as determined from the TIR to FUV flux ratio. The adopted $A(\mathrm{FUV})$ extinction correction, determined excluding the contribution of the active nucleus and extrapolating the profile to zero values in the outer disk, is shown by the dashed line. The dotted lines give the extinction profiles in the $B$ and $H$ bands. [See the electronic edition of the Journal for a color version of this figure.]

determination of the radially dependent extinction corrections, which can be quite large (i.e., more than $1 \mathrm{mag}$ ) in the UV bands, is thus the major source of systematic uncertainty in the reconstruction of the corrected radial profiles.

$\mathrm{H} \alpha+[\mathrm{N}$ II] narrowband imaging has been corrected for [ $\mathrm{N}$ II] contamination and dust extinction (Balmer decrement) using the integrated spectroscopy of Gavazzi et al. (2004). Given the nature of the integrated spectrum, these corrections are fixed and do not change with radius. We believe that such a constant correction does not introduce systematic errors in the $\mathrm{H} \alpha$ profile determination given the strongly truncated morphology of the galaxy. It is generally considered that radial effects are small for these corrections (e.g., Martin \& Kennicutt 2001). On the other hand, the integrated spectrum might be partly contaminated by the nuclear emission (the galaxy is classified as a Seyfert in NED). In any case, $[\mathrm{N}$ II] contamination and extinction are the two major sources of uncertainty in the determination of the $\mathrm{H} \alpha$ radial profile.

In Figure 3 we show all the gas and stellar profiles described above, at $1 \mathrm{kpc}$ resolution. For the surface photometry, open symbols correspond to observed values, and filled symbols correspond to extinction-corrected values. The shaded area between the two profiles graphically illustrates the magnitude of the uncertain extinction correction. We note that, although not univocally related to star formation (Boselli et al. 2004), the unextinguished $8 \mu \mathrm{m}$ image (Fig. 1) and radial profile (Fig. 4) of NGC 4569 confirm the truncated nature of the star-forming disk.

\section{MODELS}

3.1. The Multizone Models for the Chemical and Spectrophotometric Unperturbed Disk Evolution

To study the evolution of the disk of NGC 4569 at various radii, we have used the multizone (typical resolution $\sim 1 \mathrm{kpc}$ ) chemospectrophotometric models of Boissier \& Prantzos (2000), updated with an empirically determined star formation law (Boissier 

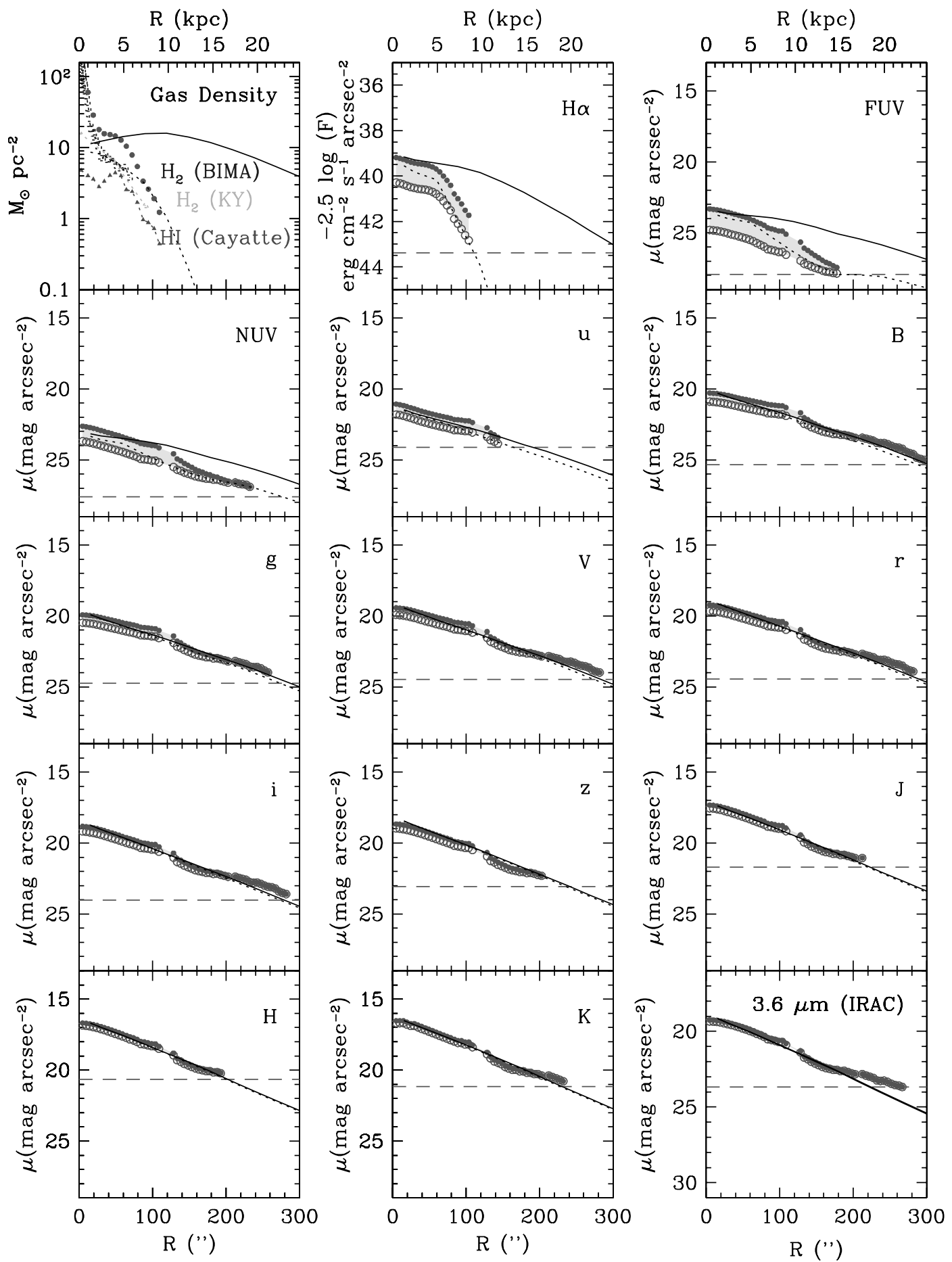

Fig. 3.-Profiles in NGC 4569. For the photometry, the open symbols are the observed values, and the filled symbols are the ones corrected for extinction. The dashed horizontal line in each panel indicates detection limits, determined as described in Gil de Paz \& Madore (2005) for a signal-to-noise ratio (S/N) $\sim 1$, where the rms is a combination of the pixel per pixel and large-scale sky background rms. The total gas profile (top left, circles) is computed from the sum of the $\mathrm{H}$ I from Cayatte et al. 1994 (triangles; labeled Cayatte), $\mathrm{H}_{2}$ from Helfer et al. (2003; labeled BIMA) and Kenney \& Young (1988; labeled KY), corrected for a helium contribution ( $\left.\times 1.4\right)$. The observations were smoothed to $1 \mathrm{kpc}$, matching the resolution used in the models. The solid and dotted lines are respectively the reference model and our best model including ram pressure (see text for details on the models). [See the electronic edition of the Journal for a color version of this figure.]

et al. 2003) relating the star formation rate to the total gas surface densities $\left(\Sigma_{\mathrm{SFR}}, \Sigma_{\text {gas }}\right)$ :

$$
\Sigma_{\mathrm{SFR}}=\alpha \Sigma_{\mathrm{gas}}^{1.48} V(R) / R
$$

where $V(R)$ is the rotation velocity at radius $R$. The resulting models are extremely similar to those in Boissier \& Prantzos
(2000) and show the same global trends. Not only do we consider the star formation law as fixed, but we also keep the same mass accretion (infall) histories as in Boissier \& Prantzos (2000), based on the assumption that before the interaction with the cluster, NGC 4569 was a "normal" spiral galaxy. The only remaining free parameters in this grid of models are the spin parameter, $\lambda$ and rotational velocity, $V_{C}$. The spin parameter is a dimensionless 


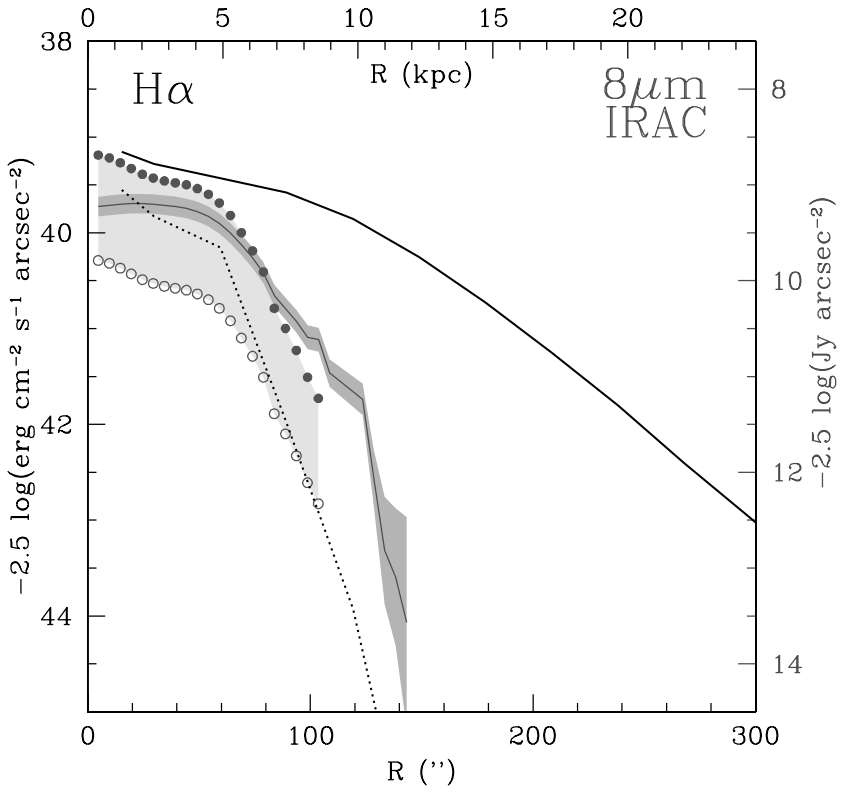

FIG. 4.-Comparison of the observed (open circles) and corrected (filled circles) $\mathrm{H} \alpha$ and $8 \mu \mathrm{m}$ IRAC (gray line) radial profiles. Both the $\mathrm{H} \alpha$ and the $8 \mu \mathrm{m}$ IRAC (gray line) radial profiles are strongly truncated if compared to the unperturbed model (solid line) and are qualitatively similar to the radial variation of the star formation activity predicted by the adopted perturbed model in a ram pressure scenario (dotted line). [See the electronic edition of the Journal for a color version of this figure.]

measure of the angular momentum (defined in, e.g., Mo et al. 1998). Its value in spiral galaxies ranges typically from $\sim 0.02$ for relatively compact galaxies to $\sim 0.09$ for low surface brightness galaxies (Boissier \& Prantzos 2000). The models of Boissier \& Prantzos (2000) contain scaling relationships (the total mass varies as $V_{C}^{3}$, and the scale length as $\left.\lambda V_{C}\right)$. Star formation histories depend on the infall timescales, which are a function of $V_{C}$ in these models, so that, roughly speaking, $V_{C}$ controls the stellar mass accumulated during the history of the galaxy, and $\lambda$ its radial distribution.

To constrain these two parameters, we use the $H$-band luminosity profile (determined assuming a distance of $17 \mathrm{Mpc}$ ) and the rotation curve of the galaxy, making the reasonable assumption that both of these observables are unperturbed during the interaction. ${ }^{8}$ Since the model does not include any bulge or nuclear component, throughout the paper the comparison between models and observed profiles is limited to the disk component, excluding the inner $\sim 40^{\prime \prime}(3 \mathrm{kpc})$. In order to fix the two parameters, we proceeded as follows: for each spin considered $(0.01-$ 0.09 in 0.01 steps), we computed models with various rotational velocities (80-360, in steps of $\left.70 \mathrm{~km} \mathrm{~s}^{-1}\right)$ and interpolated the models for each spin in order to find the velocity for which the integrated $H$-band magnitude is equal to the observed one, after 13.5 Gyr of evolution (considered the present epoch). We then compared the model profile in the $H$ band for each spin (with the velocity determined as described above) to the observed profile. The best agreement was clearly obtained with $\lambda=0.04$ and the associated $V_{C}=270 \mathrm{~km} \mathrm{~s}^{-1}$. The disk is more concentrated (extended) than observed for smaller (larger) spin parameters. The resulting rise of the rotation curve for this model is consistent with the observed one (see Fig. 5 for a comparison of this model and the constraints mentioned above). The model does not

\footnotetext{
8 The $H$-band luminosity, proportional to the total dynamical mass of the galaxy (Gavazzi et al. 1996) and tracer of the old stellar population, and the rotational velocity of galaxies are generally not affected by the interaction with the harsh cluster environment (Boselli \& Gavazzi 2006).
}

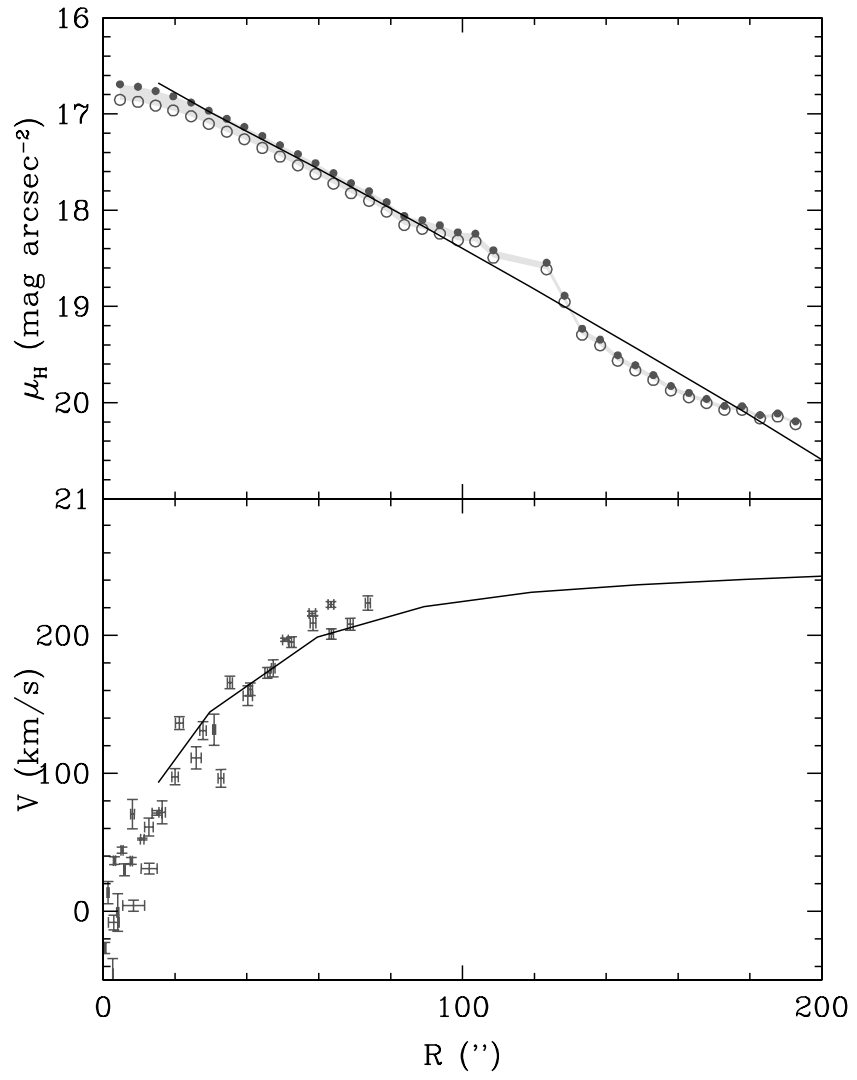

FIG. 5.-Radial profile of observed (open symbols) and extinction-corrected ( filled symbols) $\mathrm{H}$-band surface brightness (top) and of the rotational velocity (bottom) used to constrain the model without interaction (solid line). The inner $3 \mathrm{kpc}\left(40^{\prime \prime}\right)$ are not used to constrain the model. [See the electronic edition of the Journal for a color version of this figure.]

reproduce small-scale variations, probably due to structures such as spiral arms. Given the agreement, this model is now considered the reference model for the unperturbed case. The models of Boissier \& Prantzos (2000) provide the luminosity profiles in all bands as well as the total gas profile. They do not compute the nebular emission, but we estimated the $\mathrm{H} \alpha$ emission here by using the number of ionizing photons predicted by version 5 of Starburst99 (Vazquez \& Leitherer 2005) for a single generation of stars distributed on the Kroupa et al. (1993) initial mass function (as used in our models), convolving it with our star formation history and converting the result into our $\mathrm{H} \alpha$ flux following Appendix A of Gavazzi et al. (2002a). All the profiles predicted for the reference model (without any interaction, solid line) are compared to the observed one in Figure 3. The profiles at long wavelengths are in agreement with the model, while short-wavelength observations, the star formation tracers ( $\mathrm{UV}$ and $\mathrm{H} \alpha$ ), and the gas profiles present truncations and/or shorter scale lengths with respect to the expectations of the unperturbed model.

\subsection{The Starvation Scenario}

In the starvation scenario (Larson et al. 1980; Balogh et al. 2000; Treu et al. 2003), the cluster acts on large scales by removing any extended gaseous halo surrounding the galaxy, preventing further infall of such gas onto the disk. The galaxy then becomes anemic simply because it exhausts the gas reservoir through ongoing star formation.

Infall is a necessary assumption in models of the chemical evolution of the Milky Way to account for the G dwarf metallicity distribution (Tinsley 1980) and is supported by some chemodynamical 
models (Samland et al. 1997). As the disk galaxy models were obtained through a generalization of the Milky Way model, infall is present in all our models. It is a schematic but simple way to describe the growth of any galaxy from a protogalactic clump in the distant past to a massive present-day galaxy. Infall timescales in the models were chosen to reproduce the properties of presentday normal galaxies (Boissier \& Prantzos 2000; Boissier et al. 2001). A radial variation of infall timescales is suggested by dynamical models (Larson 1976). A prescription for this variation was implemented in our models of the Milky Way and of spiral galaxies, allowing us to reproduce many profiles and abundance gradients in our Galaxy (Boissier \& Prantzos 1999; Hou et al. 2000), as well as color and abundance gradients in external galaxies (Prantzos \& Boissier 2000).

Stopping infall (in order to mimic starvation) at a given time is straightforward to include in the model. We call $t_{s}$ the elapsed time since the infall termination (look-back time). In order to affect the evolution of the galaxy, starvation must have occurred before most of the gas had been accreted onto the disk. Regions where infall occurs early on with respect to the starvation epoch will barely be affected, while those where infall occurs late in the history of the galaxy will never be built up. Starvation is a global effect $\left(t_{s}\right.$ has no dependence on radius); however, the infall timescale increases with radius (inside-out formation of the galaxy). We can therefore expect an effect on the gas and stellar profiles, since starvation will affect the outer regions (since these form late in an isolated galaxy model) more than inner regions (which have already formed at earlier times).

\subsection{The Ram Pressure Stripping Scenario}

In addition to the starvation scenario we can also study the effect of ram pressure gas stripping. For simplicity, we adopt the plausible scenario of Vollmer et al. (2001b) explicitly tailored to Virgo: i.e., the galaxy being modeled has crossed the dense IGM only once, on an elliptical orbit. The ram pressure exerted by the IGM on the galaxy interstellar medium varies in time following a Gaussian profile, whose peak at $\left(t=t_{\mathrm{rp}}\right)$ is when the galaxy is crossing the dense cluster core at high velocity $\left(t\right.$ and $t_{\mathrm{rp}}$ are lookback times, where the present epoch corresponds to $t=0$ ). The Gaussian has a width $\Delta t=9 \times 10^{7} \mathrm{yr}$ (see Fig. 3 of Vollmer et al. 2001b). We make the hypothesis that the gas is removed at a rate that is directly proportional to the galaxy gas column density $\Sigma_{\text {gas }}$ and inversely proportional to the potential of the galaxy, measured by the total (baryonic) local density $\Sigma_{\text {potential }}$ (provided by the model). The gas-loss rate adopted is then equal to $\epsilon \Sigma_{\text {gas }} / \Sigma_{\text {potential }}$, with the efficiency $\epsilon$ following a Gaussian having a maximum $\epsilon_{0}$ at the time $t_{\mathrm{rp}}$, chosen to mimic the variation of the ram pressure suggested by Vollmer et al. (2001b). This very simple, but physically motivated, prescription should allow us to model the gas removal from the galaxy using only two free parameters $\left(t_{\text {rp }}\right.$ and $\left.\epsilon_{0}\right)$ to age-date and measure the magnitude of this effect. We make the further assumption that no extra star formation is induced during the interaction. This assumption is reasonable, since both models (Fujita 1998; Fujita \& Nagashima 1999) and observations (Iglesias-Paramo et al. 2004) do not show any significant increase of the star formation activity in galaxies thought to be currently undergoing a ram pressure stripping event.

\section{THE STAR FORMATION HISTORY OF NGC 4569: MODEL PREDICTIONS}

\subsection{The Starvation Scenario}

The result of starvation on the gas and star-forming ( $\mathrm{H} \alpha$ and FUV) profiles, once gas infall on the galaxy has been stopped at

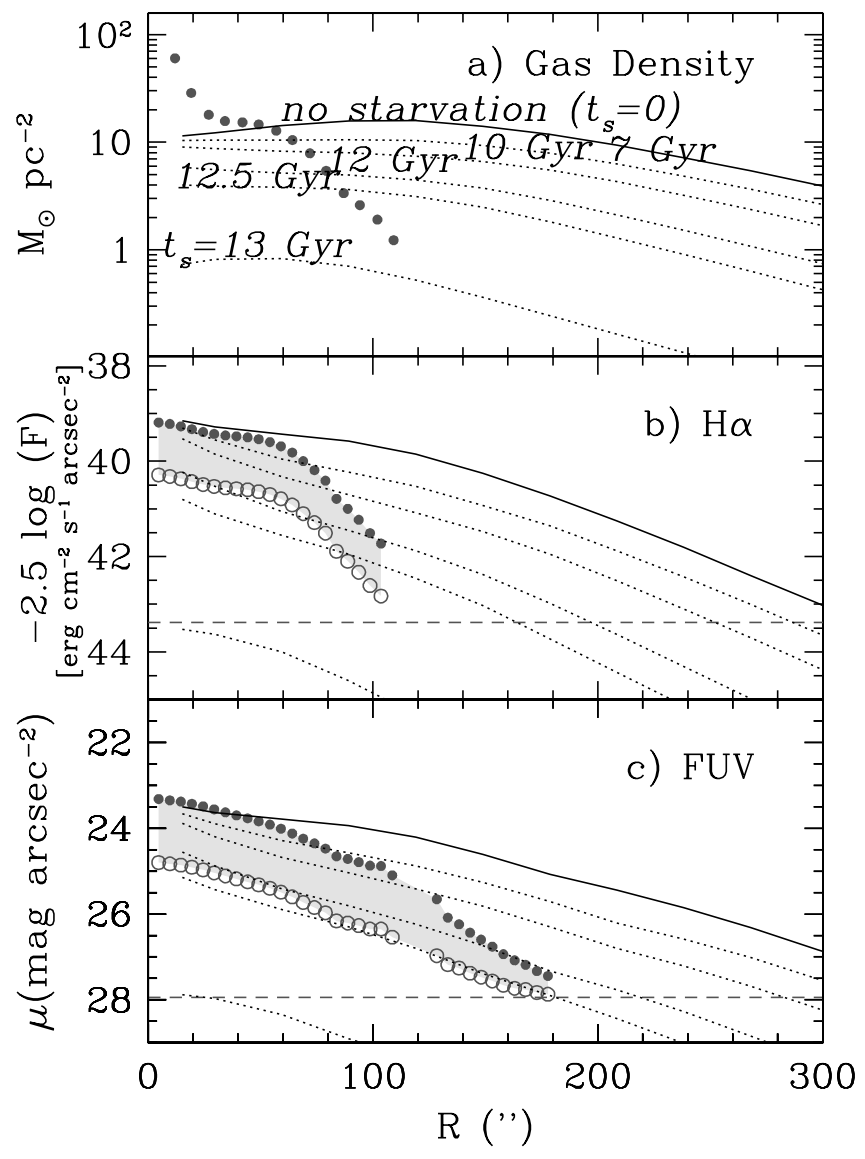

FIG. 6.-Observed (open circles) and corrected ( filled circles) (a) gas, (b) H $\alpha$, and (c) FUV profiles, compared to the predictions for the reference model (solid lines) and starvation models (in which infall has been stopped for the time $t_{s}$, indicated on top of each dotted curve). The dashed lines correspond to our detection limit. [See the electronic edition of the Journal for a color version of this figure.]

different epochs $\left(t_{s}\right)$, is shown in Figure 6. First, we note that in order to remove significant amounts of gas in N4569, infall must have been stopped for many gigayears. This is consistent with Balogh et al. (2000). The reason for this is that with $V_{C}=$ $270 \mathrm{~km} \mathrm{~s}^{-1}$, the reference model has a higher mass than the Milky Way. Since infall timescales in the models of Boissier \& Prantzos (2000) are shorter for more massive galaxies, NGC 4569 should have accreted most of its material early on in its history; thus, stopping infall at a later time has no effect, as most of the gas is already in the disk, and the galaxy is barely affected by the starvation process. Given the strong relationship between gas and star formation, the same conclusion applies to the $\mathrm{H} \alpha$ radial profile. Second, the resulting profiles never have the right shape and never predict a sharp truncation, as is observed in all the short-timescale indicators (gas, $\mathrm{H} \alpha$, and $8 \mu \mathrm{m}$ ). While the models include a radial variation of infall timescales (inside-out formation), this trend with galactocentric distance is not strong enough for the gas, $\mathrm{H} \alpha$, and FUV profiles to be truncated when infall is globally stopped at a given time.

The dependence of the infall timescale on the mass of the galaxy and on the radius are the ones encoded in the models. They would need to be dramatically changed in order to match the gas profile observed in NGC 4569 in a starvation scenario. However, if the mass dependence were extremely different, the models of Boissier \& Prantzos (2000) would fail to reproduce other relations (e.g., the observed color-magnitude relationship of nearby galaxies). The radial dependence of infall timescales in 

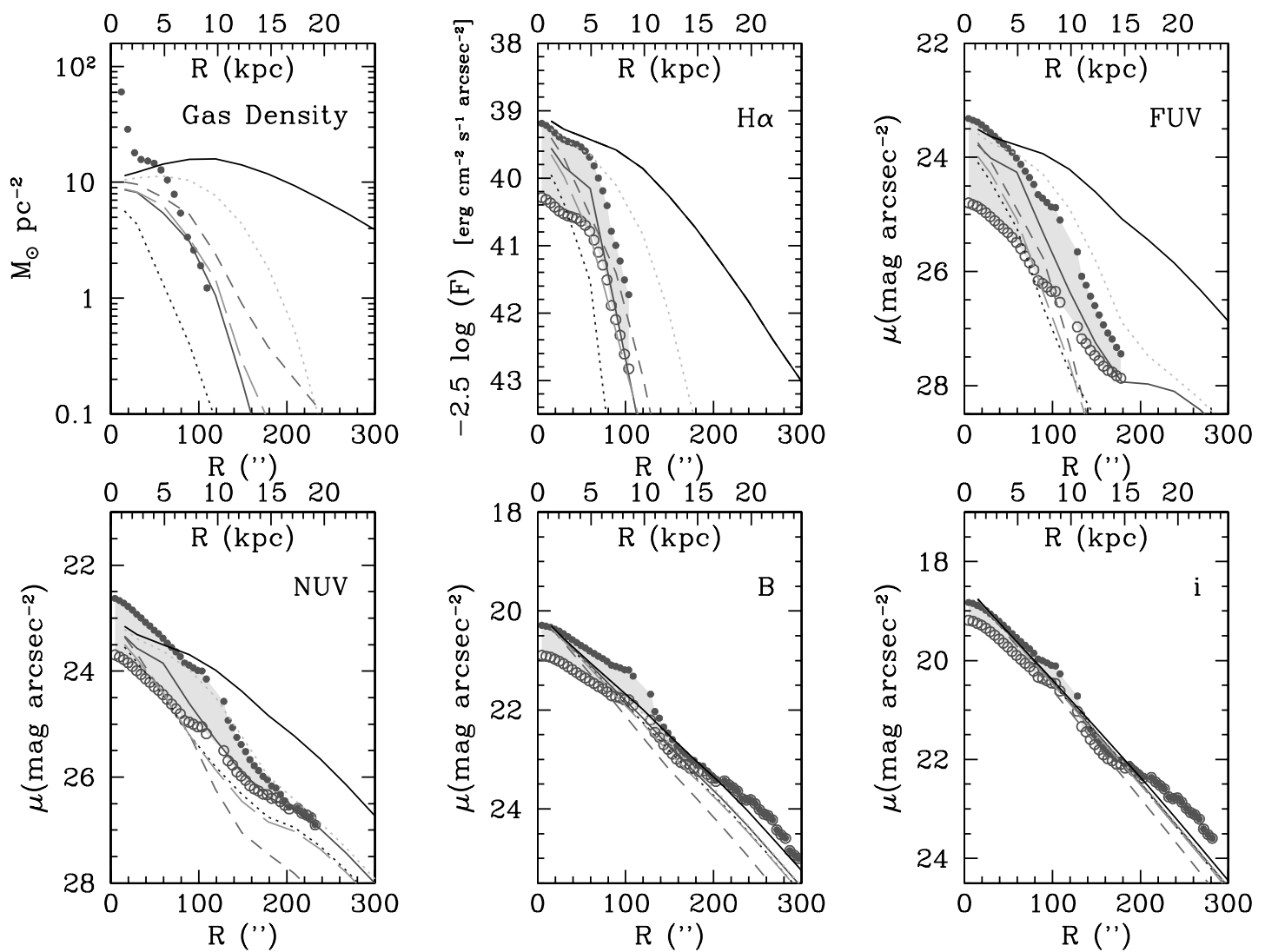

FIG. 7.- Radial profile of the observed (open circles) and extinction-corrected ( filled circles) total gas, H $\alpha$, FUV (1530 $\AA$ ), NUV (2310 $\AA$ ), $B$, and $i$ surface brightness. The shaded areas mark the range between the observed (bottom side) and extinction-corrected (top side) surface brightness profiles. Surface brightnesses are compared to the model predictions without interaction (solid lines) or with interaction (ram pressure stripping) for several $\epsilon_{0}$ and $t_{\mathrm{rp}}$ parameters. Equal maximum efficiency $\left(\epsilon_{0}=1.2 \mathrm{M}_{\odot} \mathrm{kpc}^{-2} \mathrm{yr}^{-1}\right)$ and different age: $t_{\mathrm{rp}}=100 \mathrm{Myr}$, gray continuum lines (the adopted model); $t_{\mathrm{rp}}=300 \mathrm{Myr}$, gray long-dashed lines (time suggested

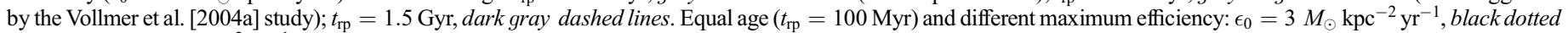
lines; $\epsilon_{0}=1 / 3 M_{\odot} \mathrm{kpc}^{-2} \mathrm{yr}^{-1}$, light gray dotted lines. [See the electronic edition of the Journal for a color version of this figure.]

these models is such that it provides a good match to the color and abundance gradients of spiral galaxies (Prantzos \& Boissier 2000). This agreement would also be broken if we drastically changed this radial trend, as it would be needed to obtain a truncated profile, similar to the one observed in NGC 4569.

\subsection{The Ram Pressure Stripping Scenario}

Having such a simple prescription with only two free parameters (as explained in $\S 3.3$ ), it is possible to choose simultaneously $t_{\mathrm{rp}}$ and $\epsilon_{0}$ because the amount of gas left and its radial distribution depend strongly on $\epsilon_{0}$, while the resulting stellar light profiles depend mainly on $t_{\mathrm{rp}}$ (see Fig. 7 for some examples).

If the time of cluster core crossing is recent, only the youngest stellar populations (emitting in $\mathrm{H} \alpha$, whose age is $\leq 4 \times 10^{6} \mathrm{yr}$, or FUV, whose age is $\leq 10^{8} \mathrm{yr}$ ) have had time to feel the progressive radial suppression of the star formation activity, and we can date the suppression of gas with our model predictions. We stress that this model is principally constrained by the radial variation of the surface brightness and color profiles and only in a minor way by their absolute values, which can be affected by zero-point uncertainties.

Among the uncertainties, the extinction corrections are certainly the largest because the $A(\mathrm{FUV})$ versus TIR/FUV calibration is highly uncertain in such evolved galaxies where dust is not only heated by UV photons but also by the general interstellar radiation field produced by more evolved stars. Geometry and attenuation laws can vary from one galaxy to another, and without a complete radiative transfer model, it is impossible to apply "perfect" extinc- tion corrections. We present in Figures 3-7 the data "as observed" (open symbols) and "extinction corrected" (filled symbols) in order to illustrate the sense and magnitude of the correction and, in doing so, illustrate just how much the uncertainty affects our results. It is obvious that allowing ourselves to change only two parameters $\left(t_{\mathrm{rp}}\right.$ and $\left.\epsilon_{0}\right)$ and considering the constraint of 16 profiles (total gas + photometry + rotation curve) does not result in a perfect match for all of them, especially taking into account the abovementioned uncertainties. Models were computed each $100 \mathrm{Myr}$ for $0 \mathrm{Myr}<t_{\mathrm{rp}}<500 \mathrm{Myr}, 200 \mathrm{Myr}$ for $0.5 \mathrm{Gyr}<t_{\mathrm{rp}}<$ $1.5 \mathrm{Gyr}$, and $1 \mathrm{Gyr}$ for $1.5 \mathrm{Gyr}<t_{\mathrm{rp}}<6.5 \mathrm{Gyr}$; and in steps of $0.2 M_{\odot} \mathrm{kpc}^{-2} \mathrm{yr}^{-1}$ efficiencies between 0.4 and 1.6. Computing the $\chi^{2}$ for various values of $\epsilon_{0}$ and $t_{\mathrm{rp}}$ (see Fig. 8), we found that the model best matching the profiles of NGC 4569 is characterized by $\epsilon_{0}=1.2 M_{\odot} \mathrm{kpc}^{-2} \mathrm{yr}^{-1}$ and $t_{\mathrm{rp}}=100 \mathrm{Myr}$. The $\chi^{2}$ for models with $t_{\mathrm{rp}}<400 \mathrm{Myr}$ are still acceptable with $\chi^{2}$ lower than 5 times the $\chi^{2}$ of the best model $\left(\chi_{\min }^{2}=3.4\right)$. The $\chi^{2}$ for models in disagreement with observational limits at large radii (e.g., predicting too much gas or light in the outer disk where none is observed) are artificially set to large values to reject these solutions that cannot reproduce the observed truncations (shaded area in Fig. 8). Note that although the reduced $\chi^{2}$ was computed, with two free parameters, the usual idea of a good fit $\left(\chi^{2} \sim 1\right)$ cannot be achieved, since we know that our models will not reproduce the small-scale variations of the profiles (as mentioned above), and the introduction of observational limits makes any statistical analysis harder. In our study, the $\chi^{2}$ is only used to pick out the best among the models, and to see which parameters give 


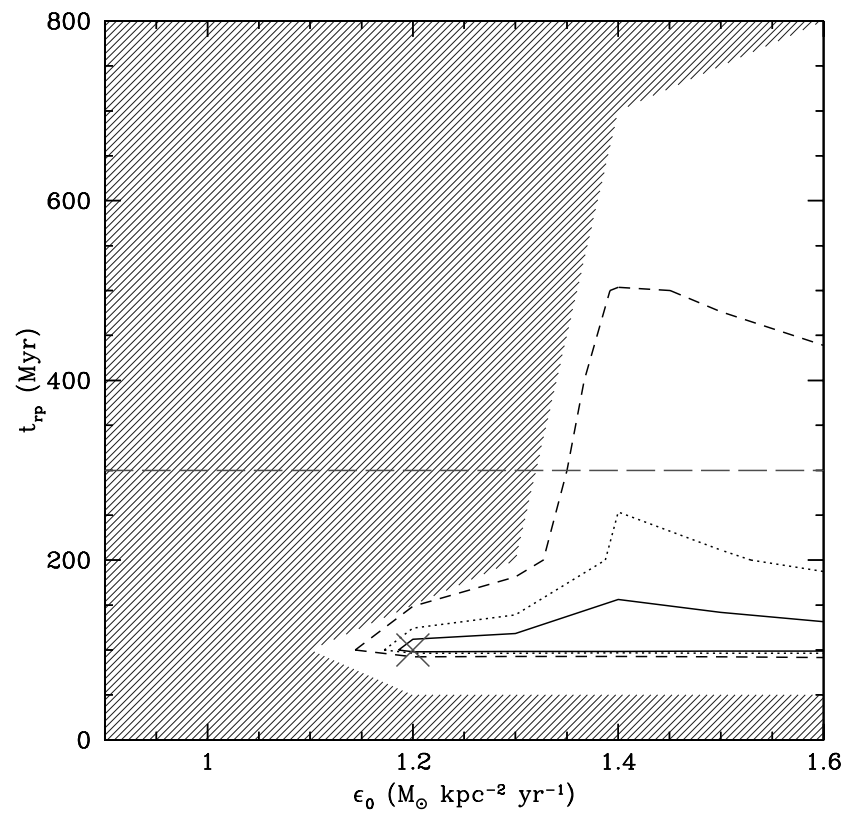

FIG. 8.- Reduced $\chi^{2}$ as a function of the look-back time of the ram pressure event $t_{\mathrm{rp}}$ and efficiency $\epsilon_{0}$. The contours (solid, dotted, and dashed) show $\chi^{2}$ levels of 2,3 , and $5 \chi_{\min }$, respectively (the best model gives a value of $\chi_{\min } \sim 3.4$; a "perfect fit" with $\chi^{2} \sim 1$ cannot be achieved, since the models will never reproduce observed small-scale variations). The horizontal long-dashed line indicates the time suggested by the dynamical model of Vollmer et al. (2004a). The shaded area indicates values for which the model surface brightnesses are in disagreement with observational limits (nondetections at relatively large radii). In this case, the $\chi^{2}$ was arbitrarily given large values in order to reject those solutions that would not remove enough gas to properly reproduce the observed truncation. [See the electronic edition of the Journal for a color version of this figure.]

similar results $\left(t_{\mathrm{rp}}<400 \mathrm{Myr}\right)$ and which parameters produce very unrealistic models (large $t_{\text {rp }}$ and low $\epsilon_{0}$ ). For a few profiles Figure 7 shows the observations contrasted with models of various ages $\left(t_{\mathrm{rp}}\right)$ and of various efficiencies, showing that low and/ or high efficiencies do not properly reproduce the gas and $\mathrm{H} \alpha$ truncation. In this figure, we also show a model with $t_{\mathrm{rp}}=300 \mathrm{Myr}$, the time indicated by the dynamical model of Vollmer et al. (2004a). It is interesting to note that, although earlier cluster core crossing epochs give more truncated disk profiles in the old stellar populations ( $B$ and $i$ bands, black dotted line), this is not the case in the gas profile, which is modified by contributions from the recycled gas. Models with $t_{\mathrm{rp}}>500 \mathrm{Myr}$ are quickly rejected because recycled gas from evolving stars would be present at large radii, thereby allowing some low levels of star formation. The model relative to the oldest interaction, $t_{\mathrm{rp}}=1.5 \mathrm{Gyr}$ given in Figure 7 (dashed line), predicts in fact a factor of $\sim 2$ higher total gas surface density outside the $80^{\prime \prime}$ radius than that observed.

This is largely consistent with the dynamical models of Vollmer et al. (2004a), who obtained $t_{\mathrm{rp}} \sim 300 \mathrm{Myr}$, but did not reject shorter ( 100 Myr) timescales (B. Vollmer 2006, private communication). Although not perfectly reproducing the surface brightness profiles, the best-fitting model is able to qualitatively reproduce the truncation of the total gas disk profile and that of the $\mathrm{H} \alpha$ and UV radial profiles, as well as the milder truncation observed at longer wavelengths (see Fig. 3). The comparison with the unperturbed model (assumed to represent an isolated spiral galaxy) clearly shows the effect of a ram pressure stripping event.

The ram pressure model also reproduces (within the uncertainties) the radial trends of colors, which are also clearly different from the unperturbed reference model. This is especially visible in Figure 9, where we have compiled a few color pro-

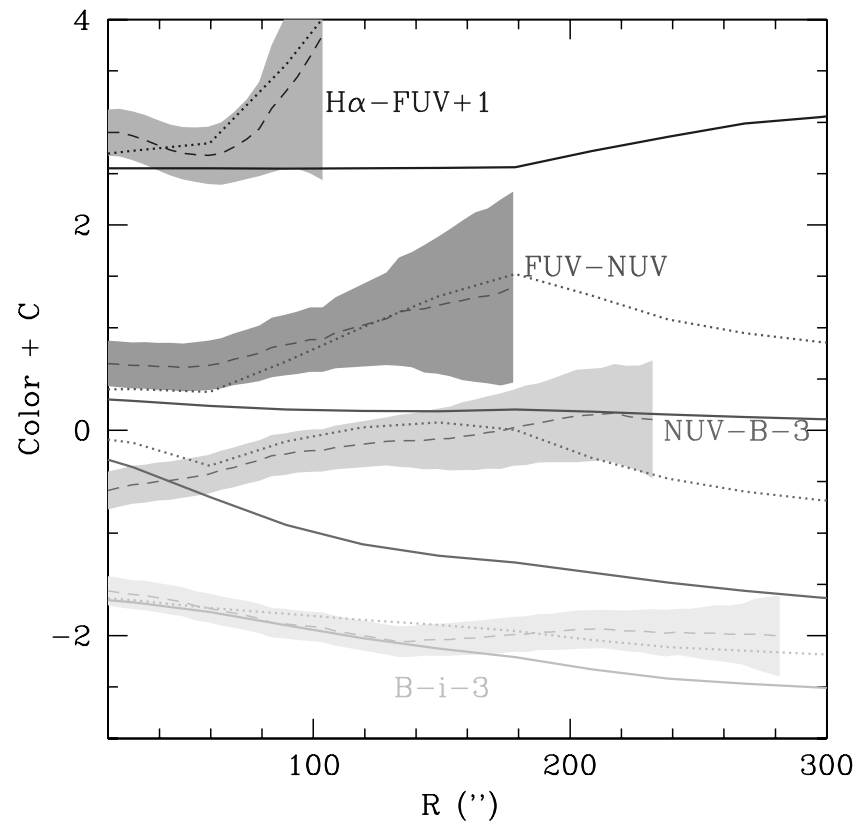

FIG. 9.--Observed color profile (dashed curves) and uncertainties (shaded areas). The solid lines are the results of the unperturbed model, while the dotted lines correspond to the best model with ram pressure. [See the electronic edition of the Journal for a color version of this figure.]

files: the observed data (smoothed to match the model resolution), the unperturbed reference model, and the model including the perturbation. Color gradients at short wavelengths are inverted (redder colors outward) with respect to normal galaxies when gas removal is introduced into the model, as observed.

The adopted model does not take into account possible effects complicating the interaction. The most obvious one is the possibility for some of the gas to be reaccreted onto the galaxy following the stripping episode. This happens in several of the dynamical models run by Vollmer et al. (2001b). The effect is strongly dependent on the inclination angle of the galaxy with respect to its orbital plane, as well as the maximum ram pressure. In all of their models having a significant inclination (i.e., larger than $20^{\circ}$ ), the reaccreted gas mass is lower than $10 \%$ of the stripped gas. If $10 \%$ of the stripped gas in our model were reaccreted (and distributed in a similar way to the gas profile we obtained), the gas profile would increase by only $0.4 \mathrm{dex}$, which would not be very different from the observed profiles. If we assume in the models that a much larger fraction of the gas (about 50\%) is reaccreted, we would obtain $\mathrm{H}$ I column densities exceeding the observed ones. Another reason why we do not expect much reaccretion to have taken place is that this phenomenon takes time: several 100 Myr after the closest passage of the galaxy to the cluster center, a time similar to the look-back time we expect for this passage.

To conclude, we can confidently say that the starvation scenario is very unlikely to have shaped the NGC 4569 profiles, while the ram pressure scenario is much more in agreement with the observations. With this scenario, we can exclude solutions older than $\sim 500 \mathrm{Myr}$, in agreement with the dynamical models of Vollmer et al. (2001b).

\section{DISCUSSION AND CONCLUSION}

The present work gives the first quantitative estimate of the structural evolution of stellar disks in cluster galaxies due to gas removal caused by a dynamical interaction of the galaxy with the IGM, delivering a strong message concerning the passive stellar 


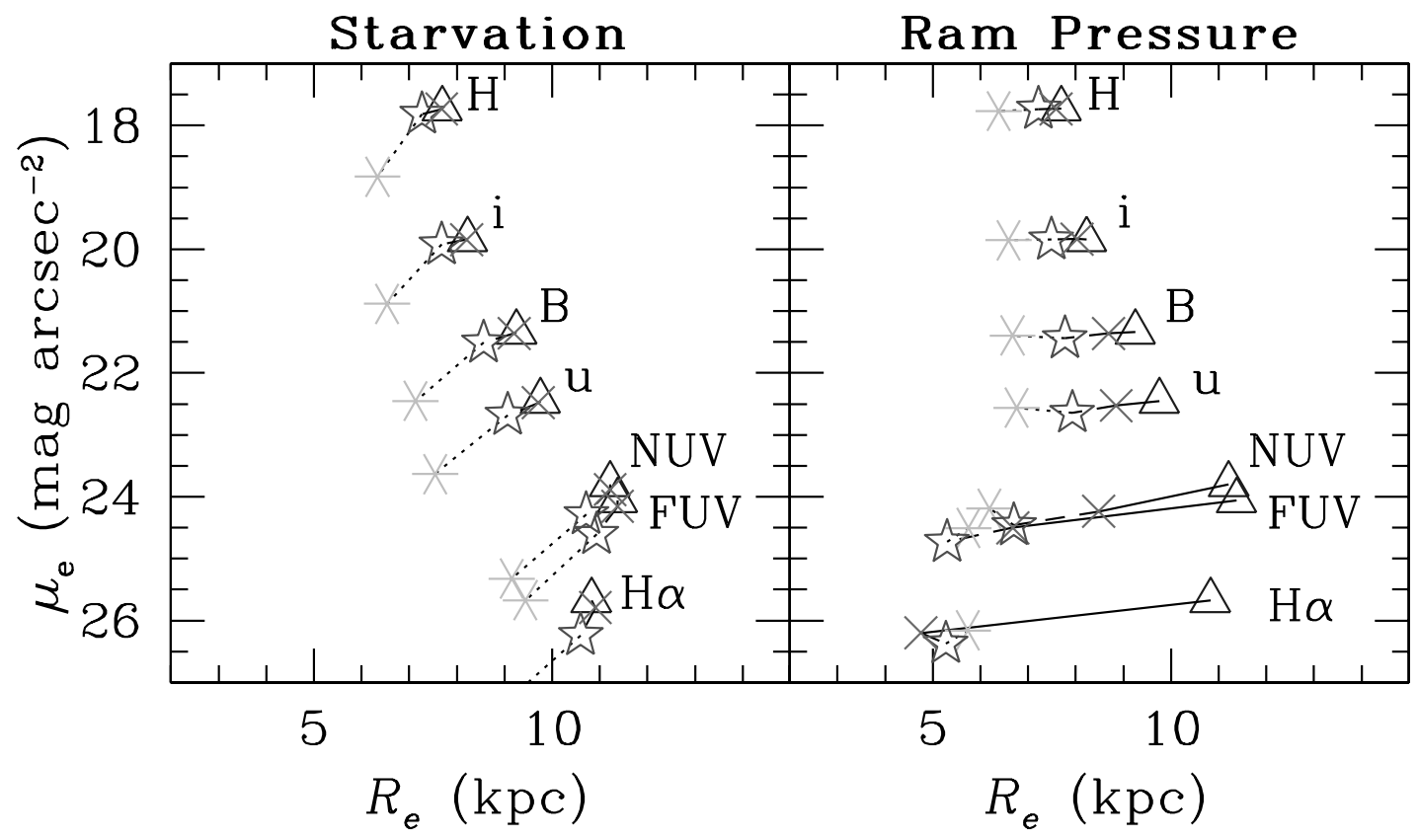

\begin{tabular}{|ll|lr|}
$\triangle$ None & $\sim \mathrm{t}_{\mathrm{s}}=7 \mathrm{Gyr}$ & $\triangle$ None $\quad \sim \mathrm{t}_{0}=1.5 \mathrm{Gyr}$ \\
$\times \mathrm{t}_{\mathrm{s}}=1 \mathrm{Gyr}$ & $\times \mathrm{t}_{\mathrm{s}}=12 \mathrm{Gyr}$ & $\times \mathrm{t}_{0}=0.1 \mathrm{Gyr} \times \mathrm{t}_{0}=5.5 \mathrm{Gyr}$ \\
\hline
\end{tabular}

FIG. 10.-Variation of the effective surface brightness (mean surface brightness within $R_{e}$, the radius containing half of the total light) and radius due to differential variation of the star formation history of NGC 4569 in (left) starvation and (right) ram pressure stripping scenarios. Open triangles are for the unperturbed model; the other symbols are for different ages of the interaction. [See the electronic edition of the Journal for a color version of this figure.]

evolution of stripped disks. First of all, it is clear that the truncation of the total gas disk profile is soon reflected on the young population stellar disk, confirming the predictions of Larson et al. (1980). As a consequence, gas-stripped galaxies have color gradients opposite to that of normal, isolated spiral galaxies, which generally have bluer colors in their outer disks. NGC 4569 is bluest toward the center (see Figs. 9 and 11). The trend is especially true for colors tracing the relatively young populations $\left(<\sim 10^{8} \mathrm{yr}\right)$; colors tracing populations older than the interaction event show the usual gradient (i.e., redder toward the center, as the $B-i$ color index). The inversion of the color gradient, observed here for the first time in a cluster galaxy, is well reproduced by our model.

The second major conclusion of the present analysis is that the perturbation that induced the truncation of the stellar disk of NGC 4569 is relatively recent event ( $\sim 100 \mathrm{Myr}$, in any case $\leq 500 \mathrm{Myr}$ ). Ram pressure is favored with respect to starvation, since the latter is simply not able to reproduce the observed truncation of the gas and star-forming ( $\mathrm{H} \alpha, 8 \mu \mathrm{m}$, and FUV) disk.

Since NGC 4569 seems to be typical of the $\mathrm{H}$ I-deficient galaxy population inhabiting nearby clusters, characterized by truncated $\mathrm{H}_{\mathrm{I}}$ and star-forming disks (Cayatte et al. 1994; Koopmann et al. 2006), with largely unperturbed older stellar populations, ${ }^{9}$ we generalize this statement by saying that gas stripping in cluster galaxies is due to relatively recent perturbations. This result is thus a major constraint on the evolution of cluster galaxies, since it rejects long-timescale phenomena such as harassment and starvation.

\footnotetext{
9 We remind the reader that there is a strong relationship between the ratio of the optical to $\mathrm{H} \alpha$ radii and the $\mathrm{H}$ I deficiency parameter in cluster galaxies (see Fig. 11 of Boselli \& Gavazzi 2006).
}

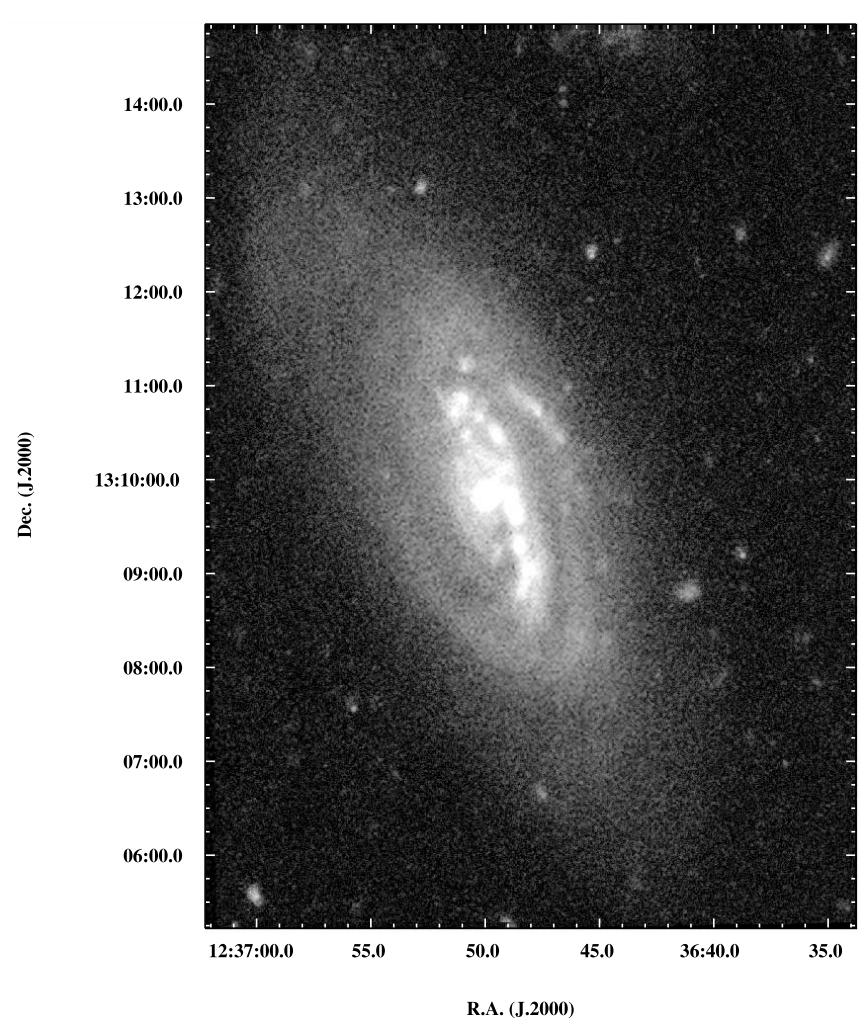

FIG. 11.-RGB (continuum-subtracted $\mathrm{H} \alpha$, FUV = white clumpy; red continuum near $\mathrm{H} \alpha=$ diffuse gray) color map of NGC 4569. The northwest spiral arm is visible at R.A. $\sim 12^{\mathrm{h}} 37^{\mathrm{m}} 47^{\mathrm{s}}$, decl. $\sim 13^{\circ} 10^{\prime} 40^{\prime \prime}$. [See the electronic edition of the Journal for a color version of this figure.] 
The ram pressure stripping scenario has recently been criticized as unable to reproduce the radial decrease with the clustercentric distance of the star formation activity of galaxies in the nearby universe. Recent, complete spectroscopic surveys of the nearby universe, such as the Sloan Digital Sky Survey or the Two Degree Field, have shown that the activity of late-type galaxies begins to decrease at 1-2 virial radii from the cluster center (Gomez et al. 2003; Lewis et al. 2002; Tanaka et al. 2004; Nichol 2004), scale lengths comparable to those observed in the $\mathrm{H} \mathrm{I}$ (Gavazzi et al. 2005, 2006b) and/or H $\alpha$ (Gavazzi et al. 2002b, 2006a) in nearby clusters. Since these distances (1-2 virial radii) are significantly larger (a factor of 5-10) than those where galaxy-IGM interactions are expected to be more efficient ( $\sim$ inside a core radius), other processes already active at the periphery of clusters (such as galaxy harassment, Moore et al. 1996; starvation, Balogh et al. 2000; or preprocessing, Fujita 2004; Cortese et al. 2006a) have been proposed to explain the radial decrease of the star formation activity with the clustercentric distance.

As extensively discussed in Boselli \& Gavazzi (2006), however, the presence of galaxies with clear signs of ongoing ram pressure stripping at the periphery of nearby clusters and the large velocity dispersion, combined with the radial orbits (Dressler 1986; Solanes et al. 2001) of the late-type galaxy population, can explain the observed decrease of the star-forming activity at large clustercentric radii in a ram pressure stripping scenario. NGC 4569, for instance, might have traveled $\sim 0.6 \mathrm{Mpc}$ ( $1 / 3$ of the virial radius) since it was stripped, a distance significantly larger than the core radius of the cluster $(130 \mathrm{kpc})$. We recall that in Virgo the drop of the star formation in bright galaxies is observed at $\sim 0.7$ virial radii (Gavazzi et al. 2006a), while the increase of the $\mathrm{H}$ I deficiency parameter occurs at about 1 virial radius (Gavazzi et al. 2005). The higher velocity dispersion and IGM gas density of rich, evolved clusters, as well as a clumpy IGM distribution, might thus be at the origin of ram pressure stripped galaxies up to $\sim 1$ virial radius.

One of the most intriguing (and still open) questions regarding the effects of the environment on the evolution of galaxies is that of the origin of lenticular galaxies and their overabundance in the centers of rich clusters. Are lenticular galaxies an independent population of galaxies formed in the primordial high-density environments, or were they spiral disks whose star formation activity had been quenched once their gas reservoir was removed by the unfavorable cluster environment? The present work has shown for the first time how a galaxy cluster-IGM interaction is able to remove most of the gas reservoir, inducing important structural modifications in the stellar disk properties. We have in fact shown that, because of the differential radial stellar evolution of spiral disks, we can expect that cluster spiral galaxies have (at least at short wavelengths) more truncated disk profiles, inverting the outer color gradient with respect to similar but unperturbed objects. The surface brightness of the disk, however, mildly decreases in $\mathrm{H} \alpha$ and in the UV bands while remaining mostly constant at longer wavelengths, even 5 Gyr after the interaction (Fig. 10). ${ }^{10}$ The differential evolution of the stellar disk due to gas stripping

\footnotetext{
${ }^{10}$ Forty percent of S0 galaxies in the Coma Cluster underwent a star formation event in their centers in the last 5 Gyr (Poggianti et al. 2001).
}

alone is thus not able to reproduce the $\sim 0.65$ mag increased central surface brightness of present-day lenticular galaxies (Dressler 1980; Boselli \& Gavazzi 2006). In the case of starvation, stellar disks are not truncated ( $R_{e}$ only slightly decreases), while surface brightnesses significantly decrease on long timescales.

Gravitational perturbations, such as tidal interactions with other galaxies (Merritt 1983), interactions with the cluster potential well (Byrd \& Valtonen 1990), or a mixture of both, must be invoked to reproduce the observed properties of nearby lenticular galaxies. As described in $\S 1$, similar conclusions have been obtained from the analysis of statistical, kinematical, structural, and spectrophotometric properties of nearby clusters.

This new study is consistent with the idea that the present evolution of late-type galaxies in clusters differs from that at earlier epochs, where late-type galaxies were mostly perturbed by dynamical interactions (preprocessing and/or galaxy harassment; Dressler 2004; Moore et al. 1996), which were able to thicken the stellar disks, thereby producing the present-day cluster lenticular galaxies.

To conclude, we can say that the combination of multifrequency observations of spatially resolved cluster galaxies combined with multizone spectrophotometric models of galaxy evolution provides an extremely useful tool to study the evolution of cluster galaxies. The results here presented for a typical $\mathrm{H}$ I-deficient, anemic Virgo Cluster galaxy and extrapolated to the whole cluster galaxy population indicate the following.

1. The gas removal due to a ram pressure stripping event can reproduce the observed radial truncation of the stellar disk stronger at shorter wavelengths. On the other hand, starvation is not able to truncate gas or stellar disks.

2. As a consequence, color gradients make cluster objects redder in the outer disk than in the inner regions and are thus inverted with respect to normal, isolated late-type galaxies.

3. This technique is useful to age-date the interaction. If NGC 4569 represents the typical $\mathrm{H}$ I-deficient cluster galaxy with a truncated $\mathrm{H} \alpha$ and $\mathrm{H}$ I disk and normal intermediate age stellar disk, then our modeling suggests that gas stripping is a relatively recent event, since it probably took place $\leq 500 \mathrm{Myr}$ ago in these systems.

4. The effective surface brightness of the stripped galaxies remains constant or even mildly decreases after the interaction.

We hope to confirm this original result in the near future once multifrequency data become available for a statistically significant sample of late-type cluster galaxies.

GALEX is a NASA Small Explorer, launched in 2003 April. We gratefully acknowledge NASA's support for construction, operation, and science analysis for the GALEX mission, developed in cooperation with the Centre National d'Etudes Spatiales (CNES) of France and the Korean Ministry of Science and Technology. We thank G. Gavazzi and N. Prantzos for their long-term collaboration in the subjects studied in this paper. S. B. thanks the CNES for its funding through GALEX-Marseille. We wish to thank B. Vollmer and J. Kenney for their valuable comments, and the GALEX SODA team for their help with the data reduction.

Boissier, S., Prantzos, N., Boselli, A., \& Gavazzi, G. 2003, MNRAS, 346, 1215

Boselli, A., \& Gavazzi, G. 2002, A\&A, 386, 124 2006, PASP, 118, 517

Boselli, A., Gavazzi, G., Donas, J., \& Scodeggio, M. 2001, AJ, 121, 753 Boselli, A., Gavazzi, G., \& Sanvito, G. 2003, A\&A, 402, 37 
Boselli, A., Lequeux, J., \& Gavazzi, G. 2002, A\&A, 384, 33 2004, A\&A, 428, 409

Boselli, A., Tuffs, R., Gavazzi, G., Hippelein, H., \& Pierini, D. 1997, A\&AS, 121,507

Buat, V., et al. 2005, ApJ, 619, L51

Byrd, G., \& Valtonen, M. 1990, ApJ, 350, 89

Cayatte, V., Kotanyi, C., Balkowski, C., \& van Gorkom, J. 1994, AJ, 107, 1003

Christlein, D., \& Zabuldoff, A. I. 2004, ApJ, 616, 192

Cortese, L., Gavazzi, G., Boselli, A., Franzetti, P., Kennicutt, R., O’Neil, K., \& Sakai, S. 2006a, A\&A, 453, 847

Cortese, L., et al. 2006b, ApJ, 637, 242

Cowie, L. L., \& Songaila, A. 1977, Nature, 266, 501

Dale, D., Helou, G., Contursi, A., Silbermann, N., \& Kolhatkar, S. 2001, ApJ, 549, 215

Dale, D., et al. 2005, ApJ, 633, 857

Dressler, A. 1980, ApJ, 236, 351

. 1986, ApJ, 301, 35

2004, in Clusters of Galaxies: Probes of Cosmological Structure and

Galaxy Evolution, ed. Mulchaey et al. (Cambridge: Cambridge Univ. Press), 206

Fujita, Y. 1998, ApJ, 509, 587 2004, PASJ, 56, 29

Fujita, Y., \& Nagashima, M. 1999, ApJ, 516, 619

Gavazzi, G., Bonfanti, C., Sanvito, G., Boselli, A., \& Scodeggio, M. 2002a, ApJ, 576, 135

Gavazzi, G., Boselli, A., Cortese, L., Arosio, I., Gallazzi, A., Pedotti, P., \& Carrasco, L. 2006a, A\&A, 446, 839

Gavazzi, G., Boselli, A., Donati, A., Franzetti, P., \& Scodeggio, M. 2003, A\&A, 400, 451

Gavazzi, G., Boselli, A., Mayer, L., Iglesias-Paramo, J., Vilchez, J. M., \& Carrasco, L. 2001, ApJ, 563, L23

Gavazzi, G., Boselli, A., Pedotti, P., Gallazzi, A., \& Carrasco, L. 2002b, A\&A, 396, 449

Gavazzi, G., Boselli, A., van Driel, W., \& O’Neil, K. 2005, A\&A, 429, 439

Gavazzi, G., Franzetti, P., Scodeggio, M., Boselli, A., \& Pierini, D. 2000, A\&A, 361, 863

Gavazzi, G., O’Neil, K., Boselli, A., \& van Driel, W. 2006b, A\&A, 449, 929

Gavazzi, G., Pierini, D., \& Boselli, A. 1996, A\&A, 312, 397

Gavazzi, G., Zaccardo, A., Sanvito, G., Boselli, A., \& Bonfanti, C. 2004, A\&A, 417, 499

Gil de Paz, A., \& Madore, B. 2005, ApJS, 156, 345

Gil de Paz, A., et al. 2006, ApJS, in press (astro-ph/0606440)

Gomez, P. L., et al. 2003, ApJ, 584, 210

Gunn, J. E., \& Gott, J. R. I. 1972, ApJ, 176, 1

Jarrett, T., Chester, T., Cutri, R., Schneider, S., \& Huchra, J. 2003, AJ, 125, 525

Jogee, S., Scoville, N., \& Kenney, J. 2005, ApJ, 630, 837

Helfer, T., et al. 2003, ApJS, 145, 259

Hinz, J. L., Rieke, G. H., \& Caldwell, N. 2003, AJ, 126, 2622

Hou, J. L., Prantzos, N., \& Boissier, S. 2000, A\&A, 362, 921
Iglesias-Paramo, J., Boselli, A., Gavazzi, G., \& Zaccardo, A. 2004, A\&A, 421, 887

Kenney, J., van Gorkom, J., \& Vollmer, B. 2004, AJ, 127, 3361

Kenney, J., \& Young, J. 1988, ApJS, 66, 261

Kennicutt, R., et al. 2003, PASP, 115, 928

Koopmann, R., Haynes, M., \& Catinella, B. 2006, AJ, 131, 716

Koopmann, R., \& Kenney, J. 2004a, ApJ, 613, 851 . 2004b, ApJ, 613, 866

Kroupa, P., Tout, C. A., \& Gilmore, G. 1993, MNRAS, 262, 545

Larson, R. B. 1976, MNRAS, 176, 31

Larson, R. B., Tinsley, B., \& Caldwell, N. 1980, ApJ, 237, 692

Lewis, I., et al. 2002, MNRAS, 334, 673

Martin, C. L., \& Kennicutt, R. C. 2001, ApJ, 555, 301

Merritt, D. 1983, ApJ, 264, 24

Mo, H. J., Mao, S., \& White, S. D. M. 1998, MNRAS, 295, 319

Moore, B., Katz, N., Lake, G., Dressler, A., \& Oemler, A. 1996, Nature, 379, 613

Nichol, R. C. 2004, in Clusters of Galaxies: Probes of Cosmological Structure and Galaxy Evolution, ed. Mulchaey et al. (Cambridge: Cambridge Univ. Press), 24 Nulsen, P. E. J. 1982, MNRAS, 198, 1007

Poggianti, B. M., et al. 2001, ApJ, 563, 118 . 2004, ApJ, 601, 197

Prantzos, N., \& Boissier, S. 2000, MNRAS, 313, 338

Rubin, V., Waterman, A., \& Kenney, J. 1999, AJ, 118, 236

Samland, M., Hensler, G., \& Theis, C. 1997, ApJ, 476, 544

Solanes, J. M., Marrique, A., Garcia-Gomez, C., Gonzales-Casado, G., Giovanelli, R., \& Haynes, M. 2001, ApJ, 548, 97

Tanaka, M., Goto, T., Okamura, S., Shimasaku, K., \& Brinkmann, J. 2004, AJ, 128,2677

Tinsley, B. M. 1980, Fundam. Cosmic Phys., 5, 287

Treu, T., Ellis, R. S., Kneib, J.-P., Dressler, A., Smail, I., Czoske, O., Oemler, A., \& Natarajan, P. 2003, ApJ, 591, 53

van den Bergh, S. 1976, ApJ, 206, 883

Vázquez, G. A., \& Leitherer, C. 2005, ApJ, 621, 695

Vollmer, B., Balkowski, C., Cayatte, V., van Driel, W., \& Huchtmeier, W. 2004a, A\&A, 419, 35

Vollmer, B., Beck, R., Kenney, J., \& van Gorkom, J. 2004b, AJ, 127, 3375

Vollmer, B., Braine, J., Balkowski, C., Cayatte, V., \& Duschl, W. 2001a, A\&A, 374,824

Vollmer, B., Cayatte, V., Balkowski, C., \& Duschl, W. 2001b, ApJ, 561, 708

Vollmer, B., Cayatte, V., Boselli, A., Balkowski, C., \& Duschl, W. 1999, A\&A, 349,411

Vollmer, B., Marcelin, M., Amram, P., Balkowski, C., Cayatte, V., \& Garrido, O. 2000, A\&A, 364, 532

Whitmore, B. C., Gilmore, D. M., \& Jones, C. 1993, ApJ, 407, 489

Yoshida, M., et al. 2004, AJ, 127, 90 